\title{
Multiple-Edge-Fault Tolerance with Respect to Hypercubes
}

\author{
Ting-Yi Sung, Men-Yang Lin, and Tung-Yang Ho
}

\begin{abstract}
Previous works on edge-fault tolerance with respect to hypercubes $Q_{n}$ are mainly focused on 1-edge fault and 2- or 3edge fault with limited size of $n$. We give a construction scheme for 2-EFT $\left(Q_{n}\right)$ graphs and 3-EFT $\left(Q_{n}\right)$ graphs, where $n$ is arbitrarily large. In our constructions, approximately $\log n$ extra degree is added to the vertices of $Q_{n}$ for 2-edge-fault tolerance, and one more degree for 3-edge-fault tolerance.
\end{abstract}

Index Terms-Edge-fault tolerance, reconfigurations, hypercubes, interconnection networks, linear algebra, vector space.

\section{INTRODUCTION}

W E usually use a graph to represent the architecture of an interconnection network, where nodes represent processors and edges represent communication links between pairs of processors. Faults may occur in nodes and/or edges of an interconnection network. We restrict faults to edges only in this paper. We first formally define edge-fault tolerance in graph terminology, which was proposed by Harary and Hayes [4]. Let $G_{1}=\left(V, E_{1}\right)$ be a graph and $G_{2}=\left(V, E_{2}\right)$ be a subgraph of $G_{1}$. We use $G_{1}-G_{2}$ or $G_{1}-E_{2}$ to denote the graph obtained from $G_{1}$ by removing all edges in $E_{2}$. Given a target graph $H=(V, E)$, let $G=\left(V, E^{\prime}\right)$ be a supergraph of $H$. G is said to be k-edge-fault-tolerant with respect to $H$, denoted by $k$-EFT $(H)$, if for any $F \subseteq E^{\prime}$ and $|F|=k, G-F$ contains a subgraph isomorphic to $H$, which is called a reconfiguration for $k$-edge fault $F$ (or simply a reconfiguration). A reconfiguration can be viewed as a relabeling of vertices of $G$ such that $G-F$ contains $H$. The graph $G^{*}$ is said to be optimal if $G^{*}$ contains the smallest number of edges among all $k$-EFT $(H)$ graphs. The target graph always represents some popular interconnection network for which many communication softwares are available. Extra edges are added to the target graph such that when edge faults occur, the network can be reconfigured to obtain the target graph.

Meshes and hypercubes are important network topologies. Edge-fault tolerant graphs with respect to meshes and hypercubes have been studied in [1], [2], [4], [6], [7]. In particular, let $Q_{n}$ be an $n$-dimensional hypercube. An optimal 1-EFT $\left(Q_{n}\right)$ graph $G^{*}$ has been proposed in [1], [4], [7] which is given by adding to $Q_{n}$ the set of edges $\{(v, \bar{v}) \mid$ for all $v\}$, where each entry in $\bar{v}$ is the complement of that in $v$. This 1-EFT $\left(Q_{n}\right)$ graph is called folded hypercube in [3]. Since we can construct optimal 1-EFT $\left(Q_{n}\right)$ graphs $G^{*}$, the question

- T.-Y. Sung and T.-Y. Ho are with the Institute of Information Science, Academia Sinica, Taipei, Taiwan, R.O.C. E-mail: tsung@iis.sinica.edu.tw.

- M.-Y. Lin is with the Department of Information Management, National Taichung Institute of Commerce, Taichung, Taiwan, R.O.C.

Manuscript received Jan. 19, 1995; revised Nov. 1, 1995.

For information on obtaining reprints of this article, please send e-mail to: transpds@computer.org, and reference IEEECS Log Number D95264. whether $k$-EFT $\left(Q_{n}\right)$ graphs for $k \geq 2$ can be derived from $G^{*}$ naturally arises. In this paper, we study multiple-edge-fault tolerance with respect to hypercube $Q_{n}$.

Bruck et al. [1] used a vector-space approach to developing 1-EFT $\left(Q_{n}\right)$ graphs with one additional wildcard dimension and used techniques from error-correcting codes to add wildcard dimensions to meshes and tori for edge-fault tolerance. They also showed that only a single wildcard dimension can be added to hypercubes. This implies that we cannot apply directly the concept of wildcard dimension to construct $k$-EFT $\left(Q_{n}\right)$ graphs for $k \geq 2$. Shih and Batcher [6] employed vector-space concept and presented necessary conditions of a so-called redundancy matrix for constructing a $k$-EFT $\left(Q_{n}\right)$ graph, which is given by the adjacency relationship of the additional edges to $Q_{n}$ (Theorem 1 in [6]). They developed an ad hoc program to generate a matrix satisfying the necessary conditions and verify its sufficiency for edge-fault tolerance. They could construct $k$ $\operatorname{EFT}\left(Q_{n}\right)$ graphs for only $k=2,3$ and $n \leq 26$ due to tremendous computation effort required in this program. We, instead, give an analytical scheme for the construction of 2-EFT $\left(Q_{n}\right)$ graphs and 3-EFT $\left(Q_{n}\right)$ graphs, where $n$ is arbitrarily large. In our constructions, the $3-\mathrm{EFT}\left(Q_{n}\right)$ graph has exactly one more edge for each vertex than in the 2-EFT $\left(Q_{n}\right)$ graph. Approximately, $\log n$ extra degree is added to $Q_{n}$ for 2-edge-fault tolerance.

\section{Definitions and Notation}

A hypercube of dimension $n$, denoted by $Q_{n}=(V, E)$, is an undirected graph consisting of $2^{n}$ vertices and $n 2^{n-1}$ edges. Each vertex $v$ has an $n$-bit binary address $v=\left(v_{1}, v_{2}, \ldots, v_{n}\right)$, where $v_{i}$ is called the $i$ th bit of $v$. We use $\bar{v}_{i}$ to denote the complement of $v_{i}$. A vertex is called the antipodal of $v$, denoted by $\bar{v}$, if $\bar{v}=\left(\bar{v}_{1}, \bar{v}_{2}, \ldots, \bar{v}_{n}\right)$. We use $v$ to denote a vertex and its corresponding row vector for binary address interchangeably. Two vertices are linked by an edge if and only if their binary addresses differ in exactly one bit. Every vertex has degree $n$. An edge $(u, v)$ is called a dimension- $i$ 
edge or simply an $i$ th edge, $1 \leq i \leq n$, if the binary addresses for $u$ and $v$ differ in the $i$ th bit. Furthermore, $u$ is called the $i$ th neighbor of $v$, denoted by $N_{i}(v)=u$. Equivalently, we have $N_{i}(u)=v$. We define $N(v)=\left\{N_{1}(v), N_{2}(v), \ldots, N_{n}(v)\right\}$. Furthermore, we also use $N(v)$ to denote the $n \times n$ matrix with row $i$ corresponding to $N_{i}(v)$.

Given a $k$-EFT $\left(Q_{n}\right)$ graph $G$, the set of edges added to $Q_{n}$ is denoted by $A$. We use $A(v)$ to denote the set of neighbors adjacent to $v$ which constitute edges in $A$. $A(v)$ is also used to represent the matrix with each row corresponding to a neighbor in $A(v)$. In a specific reconfiguration $R(F)$ for a $k$ edge fault $F$, the neighbors of $v$ are denoted by $R_{1}(v), R_{2}(v)$, $\ldots, R_{n}(v)$. Moreover, we use $R(v)$ to denote the set of $R_{1}(v)$, $\ldots, R_{n}(v)$ and the $n \times n$ matrix with the $i$ th row corresponding to $R_{i}(v)$ interchangeably. Take $Q_{3}$ and an arbitrary 1-edge fault $F=\{(001,101)\}$ as an example. We illustrate 1-EFT $\left(Q_{3}\right)$ and $R(F)$ in Fig. 1. (In Fig. 1, dotted lines represent edges in $A$.) For $v=(111), R(v)$ is given by $\{(000,111),(101,111),(110,111)\}$ or

$$
\left[\begin{array}{lll}
0 & 0 & 0 \\
1 & 0 & 1 \\
1 & 1 & 0
\end{array}\right]
$$

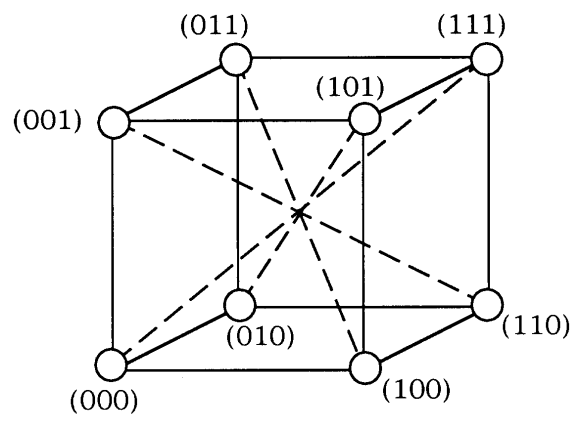

(a)

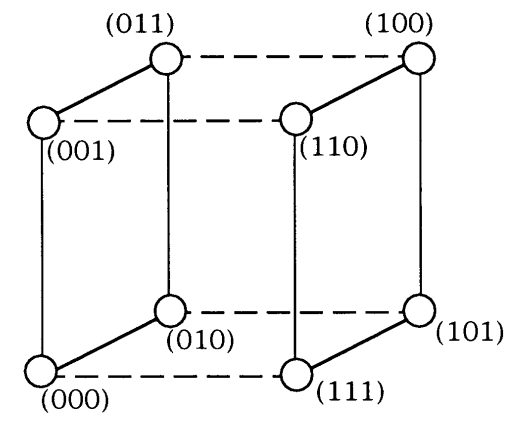

(b)

Fig. 1. For $Q_{3}$ : (a) An optimal 1-EFT $\left(Q_{3}\right)$ graph. (b) A reconfiguration for 1 -edge fault $F=\{(001,101)\}$.

For a matrix $M$, the $i$ th row and the $j$ th column of $M$ are denoted by $M_{i}$ and $M^{j}$, respectively. We use $M\left[i_{1} \ldots i_{k}, j_{1} \ldots j_{l}\right]$ to denote the $k \times l$ submatrix of $M$ that consists of rows $i_{1}$, $\ldots, i_{k}$ and columns $j_{1}, \ldots, j_{l}$. For example, the $2 \times 2$ submatrix of $A(v)$ consisting of rows $i_{1}, i_{2}$ and columns $j_{1}, j_{2}$ is denoted by $A\left[i_{1} i_{2}, j_{1} j_{2}\right](v)$ (rather than $A(v)\left[i_{1} i_{2}, j_{1} j_{2}\right]$ for clarity). The entry located at the $i$ th row and $j$ th column, $M[i, j]$, is written as $M_{i j}$.

All matrix and vector operations in this paper are defined over Galois Field GF(2). Let $v_{1}$ and $v_{2}$ be two $(0,1)$ vectors. The number of $1 \mathrm{~s}$ in $v_{1}$ is called the Hamming weight of $v_{1}$. The number of entries that are different in $v_{1}$ and $v_{2}$ is called the Hamming distance between $v_{1}$ and $v_{2}$. All edges in $Q_{n}$ have a Hamming distance of 1 .

\section{Construction of a $k$-EFT $\left(Q_{n}\right)$ Graph}

Consider a hypercube $Q_{n}$. Let $v^{0}$ denote the node $(0, \ldots, 0)$ henceforth. $N\left(v^{0}\right)$ is an identity matrix of size $n$, denoted by $I_{n}$. Let $(u, v)$ be an $i$ th edge. It follows that $u-v=e_{i}$ where $e_{i}$ is the $i$ th unit vector. Therefore, $N\left(v^{0}\right)$ can also represent address differences of all the edges incident at any specific vertex. In other words,

$$
N(v)=\underline{1} \cdot v+N\left(v^{0}\right) \quad \text { for all vertices } v,
$$

where 1 is a column vector of $1 \mathrm{~s}$. Note that $N\left(v^{0}\right)$ is invertible, i.e., rows in $N\left(v^{0}\right)$ span $G F(2)^{n}$. It follows from (1) that $N(v)-\underline{1} \cdot v$ is also invertible, i.e., rows in $N(v)-\underline{1} \cdot v$ span $G F(2)^{n}$. In this paper, we require in all reconfigurations for edge faults, $R(v)-\underline{1} \cdot v$ for each vertex has the same full rank property as in $Q_{n}$. To be specific, we aim at constructing $k$-EFT $\left(Q_{n}\right)$ graphs such that in all reconfigurations, rows in $R\left(v^{0}\right)$ span $G F(2)^{n}$, and

$$
R(v)=\underline{1} \cdot v+R\left(v^{0}\right) \quad \text { for all vertices } v \text {. }
$$

In this manner, $R\left(v^{0}\right)$ in a reconfiguration can represent address differences of the edges incident at any specific vertex. Thus, it is essential to find an invertible $R\left(v^{0}\right)$.

Based on the above discussion, we construct $k$-EFT $\left(Q_{n}\right)$ graphs using the following relationship:

$$
A(v)=A\left(v^{0}\right)+\underline{1} \cdot v \quad \text { for all } v .
$$

Our construction scheme always yields vertex-symmetric $k$-EFT $\left(Q_{n}\right)$ graphs. To find an appropriate $A\left(v^{0}\right)$, it is required that after exclusion of $k$ arbitrary rows from $\left[\begin{array}{l}N\left(v^{0}\right) \\ A\left(v^{0}\right)\end{array}\right]$, there exists an invertible $n \times n$ submatrix $R\left(v^{0}\right)$. Our construction and verification of $k$-EFT $\left(Q_{n}\right)$ graphs proceed as follows:

Step1: Construct $A\left(v^{0}\right)$.

Step2: Show that after exclusion of $k$ arbitrary rows from $\left[\begin{array}{l}N\left(v^{0}\right) \\ A\left(v^{0}\right)\end{array}\right]$, there exists an invertible $n \times n$ submatrix
$R\left(v^{0}\right)$.

Step3: A reconfiguration can be obtained according to (2).

We first state necessary conditions for $A\left(v^{0}\right)$ when an invertible $R\left(v^{0}\right)$ exists in reconfigurations for $k$-edge faults with $k \geq 2$. These necessary conditions can also be found 
in Theorem 1 of [6]; we include the proof for the sake of completeness.

LEMMA 1. When there exists an invertible $R\left(v^{0}\right), A\left(v^{0}\right)$ must satisfy the following necessary conditions:

(C-1) the Hamming distance between any two columns is at least $k-1$, and

(C-2) each column has a Hamming weight at least $k$.

PROOF. Suppose that the Hamming distance between two columns of $A\left(v^{0}\right)$, say $A^{i}\left(v^{0}\right)$ and $A^{j}\left(v^{0}\right)$, is $k-2$. Let $i_{1}, i_{2}, \ldots, i_{k-2}$ be the rows that $A^{i}\left(v^{0}\right)$ and $A^{j}\left(v^{0}\right)$ differ. Let $F_{1}$ be a $k$-edge fault given by $F_{1}=\left\{\left(v^{0}, N_{i}\left(v^{0}\right)\right)\right.$, $\left.\left(v^{0}, N_{j}\left(v^{0}\right)\right)\right\} \cup\left\{\left(v^{0}, A_{i_{p}}\left(v^{0}\right)\right) \mid 1 \leq p \leq k-2\right\}$. Removing these $k-2$ rows from $A\left(v^{0}\right)$, column $i$, and column $j$ in the reduced matrix become identical. Thus, the reconfiguration for $F_{1}$ fails since we can not obtain an invertible $R\left(v^{0}\right)$. Suppose that there is a column of $A\left(v^{0}\right)$, say $A^{j}\left(v^{0}\right)$, having only $k-1$ entries of ones. These $k-$ 1 entries are denoted by $A_{i, j}\left(v^{0}\right), 1 \leq l \leq k-1$. Consider a $k$-edge fault $F_{2}=\left\{\left(v^{0}, N_{j}\left(v^{0}\right)\right)\right\} \cup\left\{\left(v^{0}, A_{i_{p}}\left(v^{0}\right)\right) \mid 1 \leq p\right.$ $\leq k-2\}$. The reconfiguration for $F_{2}$ fails since $R^{j}\left(v^{0}\right)$ in any reconfiguration is a zero vector which is a contradiction. Hence, the lemma follows.

Necessary conditions provide guideline for finding an appropriate $A\left(v^{0}\right)$ which is sufficient for the existence of an invertible $R\left(v^{0}\right)$. In the following analysis, for a positive integer $r$, we define $m=\sum_{i=2}^{r}\left(\begin{array}{l}r \\ i\end{array}\right)$ which denotes the maximum dimension $n$ of $Q_{n}$ such that extra degree $r$ suffices to construct 2-EFT $\left(Q_{n}\right)$ and 3-EFT $\left(Q_{n}\right)$ graphs. Next, we define an $r \times m$ matrix $M_{r}$ as follows: Each column is distinct from others, and each of columns $\sum_{i=2}^{l-1}\left(\begin{array}{l}r \\ i\end{array}\right)+1$ to $\sum_{i=2}^{l}\left(\begin{array}{l}r \\ i\end{array}\right)$ contains exactly $l$ entries of $1 \mathrm{~s}$, where $2 \leq l \leq r$, and by convention $\sum_{i=2}^{l-1}\left(\begin{array}{l}r \\ i\end{array}\right)=0$ for $l=2$. To be specific, we illustrate $M_{r}$ for $r=4$. When $r=4$, we have $m=11$ and that $M_{4}$ is a $4 \times 11$ matrix given by

$$
M_{4}=\left[\begin{array}{lllllllllll}
1 & 1 & 1 & 0 & 0 & 0 & 1 & 1 & 1 & 0 & 1 \\
1 & 0 & 0 & 1 & 1 & 0 & 1 & 1 & 0 & 1 & 1 \\
0 & 1 & 0 & 1 & 0 & 1 & 1 & 0 & 1 & 1 & 1 \\
0 & 0 & 1 & 0 & 1 & 1 & 0 & 1 & 1 & 1 & 1
\end{array}\right] .
$$

Note that each column of $M_{r}$ has a Hamming weight at least 2 and that any two columns of $M_{r}$ have a Hamming distance at least 1 . Thus, $M_{r}$ satisfies (C-1) and (C-2) with $k=2$. For $Q_{n}$, we determine a minimum $r$ such that

$$
\sum_{i=2}^{r}\left(\begin{array}{l}
r \\
i
\end{array}\right) \geq n
$$

For example, when $5 \leq n \leq 11$, we have $r=4$, and $r=5$ for
$12 \leq n \leq 26$.

From now on, we use $G$ to denote the $k$-EFT $\left(Q_{n}\right)$ graphs constructed according to (1) and (3).

\section{A 2-EFT $\left(Q_{n}\right)$ GRAPH}

To construct a 2-EFT $\left(Q_{n}\right)$ graph, we find $A\left(v^{0}\right)$ from $M_{r}$ with $r$ being appropriately chosen as stated before. We arbitrarily choose $n$ columns of $M_{r}$ to define $A\left(v^{0}\right)$. In this construction, $r$ is the extra degree added to each vertex in $Q_{n}$. Since $M_{r}$ satisfies (C-1) and (C-2), $A\left(v^{0}\right)$ also satisfies (C-1) and $(\mathrm{C}-2)$. On the other hand, we will show in the following lemma that $A\left(v^{0}\right)$ is sufficient for 2-edge-fault tolerance.

LEMMA 2. Let $A\left(v^{0}\right)$ be given as above. Then, after exclusion of two arbitrary rows from $\left[\begin{array}{l}N\left(v^{0}\right) \\ A\left(v^{0}\right)\end{array}\right]$, there exists an $n \times n$ invertible submatrix $R\left(v^{0}\right)$.

Proof. If the deleted rows are in $A\left(v^{0}\right)$, then $R\left(v^{0}\right)=N\left(v^{0}\right)$. Now consider that row $i$ of $N\left(v^{0}\right)$ and row $j$ of $A\left(v^{0}\right)$ are deleted. Choose a row $\alpha \neq j$ such that $A_{\alpha i}\left(v^{0}\right)=1$; such a row $\alpha$ exists since $A^{i}\left(v^{0}\right)$ has a Hamming weight at least 2 . Define

$$
R\left(v^{0}\right)=\left[\begin{array}{cc}
I_{n-1} & 0 \\
A_{\alpha}^{\prime} & 1
\end{array}\right]
$$

after column permutation, where $A_{\alpha}\left(v^{0}\right)$ is written as $\left\lfloor A_{\alpha}^{\prime} 1\right\rfloor$ correspondingly. Thus, $R\left(v^{0}\right)$ is invertible.

Finally, consider two arbitrary rows, say rows $i_{1}$ and $i_{2}$, are deleted from $N\left(v^{0}\right)$. Since $A\left(v^{0}\right)$ satisfies (C-1) and (C-2), we can always find two rows, say rows $\alpha_{1}$ and $\alpha_{2}$, such that the $2 \times 2$ submatix $A\left[\alpha_{1} \alpha_{2}\right.$, $\left.i_{1} i_{2}\right]\left(v^{0}\right)$ is invertible. Thus,

$$
\left.R\left(v^{0}\right)=\left[\begin{array}{lc}
I_{n-2} & 0 \\
A^{\prime} & A\left[\alpha_{1} \alpha_{2}, i_{1} i_{2}\right]
\end{array}\right]\left(v^{0}\right)\right]
$$

is invertible, where

$$
\left[\begin{array}{l}
A_{\alpha_{1}}\left(v^{0}\right) \\
A_{\alpha_{2}}\left(v^{0}\right)
\end{array}\right]
$$

is written as $\left[A^{\prime} A\left[\alpha_{1} \alpha_{2}, i_{1} i_{2}\right]\left(v^{0}\right)\right]$ after column permutation. This completes the proof.

Following the proof of Lemma 2, we can obtain reconfigurations for any 2-edge fault. Furthermore, $R(v)-\underline{1} \cdot v$ spans $G F(2)^{n}$ for all $v$ in these reconfigurations. Thus, we conclude the above discussion in the following theorem.

THEOREM 1. $G$ is $2-E F T\left(Q_{n}\right)$ such that $R(v)-\underline{1} \cdot v$ spans $G F(2)^{n}$ for all $v$ in reconfigurations for any 2-edge fault.

Since the extra degree $r$ is the minimum integer satisfying (4), our proposed $A\left(v^{0}\right)$ contains the least number of 
TABLE 1

EXtra DEGREE IN 2-EFT $\left(Q_{n}\right)$ GRAPHS

\begin{tabular}{|c|c|c|c|c|c|c|c|c|c|c|c|c|c|c|}
\hline $\max n$ & 1 & 4 & 11 & 26 & 57 & 120 & 247 & 502 & 1,013 & 2,036 & 4,083 & 8,178 & 16,369 & 32,752 \\
\hline$r$ & 2 & 3 & 4 & 5 & 6 & 7 & 8 & 9 & 10 & 11 & 12 & 13 & 14 & 15 \\
\hline
\end{tabular}

rows (neighbors) under the proposed framework. We summarize in Table 1 the extra degree $r$ in our constructed 2-EFT $\left(Q_{n}\right)$ graphs for various $n$. It follows from the definition of the $r \times m$ matrix $M_{r}$ that $m=\max n=2^{r}-r-1$. Therefore, we have the following remark.

REMARK. The extra degree $r$ is approximately $\log n$ for $Q_{n}$, and $\log n+1$ will suffice. (Actually, $r \approx \log n+\log \left(1+\frac{\log n}{n}\right)$, to be more precise.)

In the following, we use an example of a 2-EFT $\left(Q_{5}\right)$ graph for illustration. It can be easily seen that $r=4$, and we choose

$$
A\left(v^{0}\right)=\left[\begin{array}{lllll}
1 & 1 & 1 & 0 & 0 \\
1 & 0 & 0 & 1 & 1 \\
0 & 1 & 0 & 1 & 0 \\
0 & 0 & 1 & 0 & 1
\end{array}\right] .
$$

The proposed 2-EFT $\left(Q_{n}\right)$ graph has the following four extra neighbors for each vertex $v: v+(11100), v+(10011), v+$ (01010), and $v+(00101)$, denoted by $A_{1}(v), A_{2}(v), A_{3}(v)$, and $A_{4}(v)$, respectively. We use the following faults to illustrate its 2-edge-fault tolerance.

- Let $F_{1}=\{(11001,11101),(10101,10001)\}$ be a 2-edge fault. We can choose the edges $\left(v, A_{1}(v)\right)$ or the edges $\left(v, A_{4}(v)\right)$ to replace all of the third edges.

- Let $F_{2}=\{(11001,11101),(10111,11111)\}$ be a 2-edge fault. We use the edges $\left(v, A_{1}(v)\right)$ and $\left(v, A_{3}(v)\right)$ to replace all of the second and the third edges.

- Let $F_{3}=\{(11001,11101),(00101,11001)\}$ be a 2-edge fault. $F_{3}$ consists of a third edge and a $\left(v, A_{1}(v)\right)$ edge. We use the edges $\left(v, A_{4}(v)\right)$ to replace all of the third edges.

\section{A 3-EFT $\left(Q_{n}\right)$ GRAPH}

Let $T_{r+1}$ be an $(r+1) \times m$ matrix, where

$$
m=\sum_{i=2}^{r}\left(\begin{array}{l}
r \\
i
\end{array}\right)
$$

given as follows:

$$
T_{r+1}=\left[\begin{array}{c}
M_{r} \\
t
\end{array}\right],
$$

where $t$ is a row vector with an entry of one if the corresponding column in $M_{r}$ has an even Hamming weight, and zero otherwise. For example, $T_{5}$ is a $5 \times 11$ matrix given as follows:

$$
T_{5}=\left[\begin{array}{c}
M_{4} \\
t
\end{array}\right]=\left[\begin{array}{lllllllllll}
1 & 1 & 1 & 0 & 0 & 0 & 1 & 1 & 1 & 0 & 1 \\
1 & 0 & 0 & 1 & 1 & 0 & 1 & 1 & 0 & 1 & 1 \\
0 & 1 & 0 & 1 & 0 & 1 & 1 & 0 & 1 & 1 & 1 \\
0 & 0 & 1 & 0 & 1 & 1 & 0 & 1 & 1 & 1 & 1 \\
1 & 1 & 1 & 1 & 1 & 1 & 0 & 0 & 0 & 0 & 1
\end{array}\right]
$$

The choice of $r$ also follows as stated before. We add $r+1$ edges to each vertex, and $A\left(v^{0}\right)$ is given by any $n$ columns of $T_{r+1}$.

LEMMA 3. $A\left(v^{0}\right)$ satisfies (C-1) and (C-2) with $k=3$.

Proof. We first show $T_{r+1}$ satisfies (C-1) and (C-2) with $k=3$. For those columns of $M_{r}$ having a Hamming weight of 2 , the corresponding entries in vector $t$ are 1s. Furthermore, since each column of $M_{r}$ has a Hamming weight at least 2, each column of $T_{r+1}$ has a Hamming weight at least 3 . When two columns of $M_{r}$ have a Hamming distance of 1 , the two corresponding entries in $t$ are different. Furthermore, since the Hamming distance between any two columns in $M_{r}$ is at least 1 , any two columns in $T_{r+1}$ have a Hamming distance at least 2. Therefore, $T_{r+1}$ satisfies (C-1) and (C-2) with $k=3$. Since $A\left(v^{0}\right)$ is obtained by choosing $n$ columns of $T_{r+1}, A\left(v^{0}\right)$ also satisfies (C-1) and (C-2) with $k=3$.

On the other hand, we will show in the next lemma that the construction of $A\left(v^{0}\right)$ is also sufficient for 3-edge-fault tolerance.

LEMMA 4. Let $A\left(v^{0}\right)$ be given as above. Then, after exclusion of at most three arbitrary rows from $\left[\begin{array}{l}N\left(v^{0}\right) \\ A\left(v^{0}\right)\end{array}\right]$, there exists an $n \times n$ invertible submatrix $R\left(v^{0}\right)$.

Proof. Since $A\left(v^{0}\right)$ satisfies (C-1) and (C-2), the existence of an invertible $R\left(v^{0}\right)$ is obvious when those deleted rows contain at most two rows of $N\left(v^{0}\right)$. We need to consider only the case that exactly three rows of $N\left(v^{0}\right)$ are deleted. Let rows $i_{1}, i_{2}$, and $i_{3}$ be deleted from $N\left(v^{0}\right)$. Let $S(j)=\left\{i \mid A_{i j}\left(v^{0}\right)=1\right.$ for $\left.1 \leq i \leq r\right\}$, and let $S=$ $S\left(i_{1}\right) \cup S\left(i_{2}\right) \cup S\left(i_{3}\right)$. Since $A\left[S, i_{1} i_{2} i_{3}\right]\left(v^{0}\right)$ is a submatrix of $M_{r}$ and satisfies (C-1) and (C-2) with $k=2$, it follows that $|S| \geq 3$. Furthermore, the $|S| \times 3$ submatrix $A\left[S, i_{1} i_{2} i_{3}\right]\left(v^{0}\right)$ has rank of at least 2 since, otherwise, the three columns of $A\left[S, i_{1} i_{2} i_{3}\right]\left(v^{0}\right)$ are identical which is a contradiction. We distinguish the following two cases:

1) $A\left[S, i_{1} i_{2} i_{3}\right]\left(v^{0}\right)$ has rank of three.

We simply choose any three rows from $A\left[S, i_{1} i_{2} i_{3}\right]\left(v^{0}\right)$, say $\alpha_{1}, \alpha_{2}$, and $\alpha_{3}$, that are linearly independent. 
Define

$$
R\left(v^{0}\right)=\left[\begin{array}{lc}
I_{n-3} & 0 \\
A^{\prime} & A\left[\alpha_{1} \alpha_{2} \alpha_{3}, i_{1} i_{2} i_{3}\right]\left(v^{0}\right)
\end{array}\right]
$$

after column permutation. Since $A\left[S, i_{1} i_{2} i_{3}\right]\left(v^{0}\right)$ is invertible, $R\left(v^{0}\right)$ is also invertible.

2) $A\left[S, i_{1} i_{2} i_{3}\right]\left(v^{0}\right)$ has rank of two.

In this case, vector $t$, i.e., $A_{r+1}\left(v^{0}\right)$ must be incorporated with $A\left[S, i_{1} i_{2} i_{3}\right]\left(v^{0}\right)$ to find an invertible $R\left(v^{0}\right)$. We first choose any two rows from $A\left[S, i_{1} i_{2} i_{3}\right]\left(v^{0}\right)$, say $\alpha_{1}$ and $\alpha_{2}$, that are linearly independent. We next show that

$$
B=\left[\begin{array}{l}
A\left[\alpha_{1} \alpha_{2}, i_{1} i_{2} i_{3}\right]\left(v^{0}\right) \\
A\left[r+1, i_{1} i_{2} i_{3}\right]\left(v^{0}\right)
\end{array}\right]
$$

is invertible. Since $A\left[S, i_{1} i_{2} i_{3}\right]\left(v^{0}\right)$ has rank of 2, $A\left[S, i_{1} i_{2} i_{3}\right]\left(v^{0}\right)$ can be reduced to a $3 \times 3$ matrix, called $C$, after eliminating identical rows. Since there is no zero row in $C$ and each column of $C$ has a Hamming weight at least 2, the matrix $C$ has the form

$$
\left[\begin{array}{lll}
1 & 1 & 0 \\
1 & 0 & 1 \\
0 & 1 & 1
\end{array}\right]
$$

after column permutation. Therefore, each row in $A\left[S, i_{1} i_{2} i_{3}\right]\left(v^{0}\right)$ has a Hamming weight of 2 since rows in $A\left[S, i_{1} i_{2} i_{3}\right]\left(v^{0}\right)$ but not in $C$ are identical to rows in $C$. Let $S T=\sum_{l=1}^{3}\left|S\left(i_{l}\right)\right|$. It follows that $S T$ is even. That is, either all of $S\left(i_{l}\right)$ are even or two of $S\left(i_{l}\right)$ are odd and one is even. As a result $A[r+1$, $\left.i_{1} i_{2} i_{3}\right]\left(v^{0}\right)$, is a row vector of ones in the former and a unit vector in the latter. In both cases, $B$ is invertible. Define $R\left(v^{0}\right)=\left[\begin{array}{cc}I_{n-3} & 0 \\ B^{\prime} & B\end{array}\right]$ after column permutation. Therefore, $R\left(v^{0}\right)$ is invertible. Hence, the lemma follows.

We conclude the above analysis in the following theorem.

THEOREM 2. $G$ is $3-E F T\left(Q_{n}\right)$ such that $R(v)-\underline{1} \cdot v$ spans $G F(2)^{n}$ for all $v$ in reconfigurations for any 3-edge fault.

$A\left(v^{0}\right)$ for 3-edge-fault tolerance is constructed from $A\left(v^{0}\right)$ for 2-edge-fault tolerance with one additional edge for each vertex. Since the 2-EFT $\left(Q_{n}\right)$ graph constructed in Section 4 contains the least number of edges under the proposed framework, the 3-EFT $\left(Q_{n}\right)$ graph constructed in this section is also minimal in the same sense.

\section{Discussion}

Constructions of $k$-EFT $\left(Q_{n}\right)$ for $k \geq 4$ are still what we seek for. Let $A$ be an $r \times n$ submatrix of $M_{r}$. For ease of exposition, we choose $A$ to be the first $n$ columns of $M_{r}$. Let $\tau$ be a permutation on $\{1,2, \ldots, n\}$. We define $A_{1}=A$ and $A_{2}$ as the matrix obtained from $A$ with columns arranged according to $\tau$. Let $D$ be a $2 r \times n$ matrix given by $D=\left[\begin{array}{l}A_{1} \\ A_{2}\end{array}\right]$, and $D^{\prime}=\left[\begin{array}{c}D \\ t\end{array}\right]$ where $t$ is the row vector as defined for $T_{r+1}$ in Section 5. Let $S_{1}(j)=\left\{l \mid A_{l j}=1\right\}$ and $S_{2}(j)=\left\{l \mid A_{l \tau(j)}=1\right\}$. We use $\Delta$ to denote the symmetric difference between two sets, e.g., $S_{1}\left(j_{1}\right) \Delta S_{1}\left(j_{2}\right)=\left(S_{1}\left(j_{1}\right)-S_{1}\left(j_{2}\right)\right) \cup\left(S_{1}\left(j_{2}\right)-S_{1}\left(j_{1}\right)\right)$. To construct a 4 -EFT $\left(Q_{n}\right)$, we restrict $\tau$ to satisfy the following conditions.

- If $\left|S_{1}\left(j_{1}\right) \Delta S_{1}\left(j_{2}\right)\right|=1$, then $\left|S_{2}\left(\tau\left(j_{1}\right)\right) \Delta S_{2}\left(\tau\left(j_{2}\right)\right)\right| \geq 2$.

- If $\left|S_{2}\left(\tau\left(j_{1}\right)\right) \Delta S_{2}\left(\tau\left(j_{2}\right)\right)\right|=1$, then $\left|S_{1}\left(j_{1}\right) \Delta S_{1}\left(j_{2}\right)\right| \geq 2$.

It follows that $D$ and $D^{\prime}$ satisfy (C-1) and (C-2) for $k=4$ and $k=5$, respectively. We have the following conjecture:

CONJECTURE 1 . The graphs constructed according to $A\left(v^{0}\right)=D$ for $k=4$ and $A\left(v^{0}\right)=D^{\prime}$ for $k=5$ are $4-E F T\left(Q_{n}\right)$ and 5-EFT $\left(Q_{n}\right)$, respectively.

It is hard to show that $D$ and $D^{\prime}$ are also sufficient for edgefault tolerance. Furthermore, characterizing such a permutation $\tau$ is also difficult.

\section{ACKNOWLEDGMENTS}

The authors would like to thank the anonymous referees for their helpful and constructive comments.

This work was partially supported by the National Science Council, Republic of China, under grant NSC-84-2213E009-009.

\section{REFERENCES}

[1] J. Bruck, R. Cypher, and C.T. Ho, "Wildcard Dimensions, Coding Theory, and Fault Tolerant Meshes and Hypercubes," IEEE Trans. Computers, vol. 44, no. 1, pp. 150-155, Jan. 1995.

[2] R.S. Chou and L.H. Hsu, "1-Edge Fault-Tolerant Design for Meshes," Parallel Processing Letters, vol. 4, no. 4, pp. 385-389, 1994.

[3] A. El-Amawy and S. Latifi, "Properties and Performance of Folded Hypercubes," IEEE Trans. Parallel and Distributed Systems, vol. 2, no. 1, pp. 31-42, Jan. 1991.

[4] F. Harary and J.P. Hayes, "Edge Fault Tolerance in Graphs," Networks, vol. 23, pp. 135-142, 1993.

[5] M. O'Nan and H. Enderton, Linear Algebra, third edition. Orlando, Fla.: Harcourt Brace Jovanovich, 1990.

[6] C.J. Shih and K.E. Batcher, "Adding Multiple-Fault Tolerance to Generalized Cube Networks," IEEE Trans. Parallel and Distributed Systems, vol. 5, no. 8, pp. 785-792, Aug. 1994.

[7] S. Ueno, A. Bagchi, S.L. Hakimi, and E.F. Schmeichel, "On Minimum Fault-Tolerant Networks," SIAM J. Disc. Mathematics, vol. 6, pp. 565-574, 1993. 


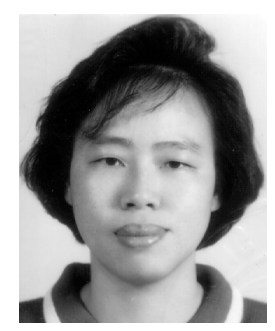

Ting-Yi Sung received her $\mathrm{PhD}$ degree in operations research from New York University in 1989. She is currently an associate research fellow at the Institute of Information Science, Academia Sinica, Taiwan, Republic of China. Her research interests include mathematical programming, graph algorithms, and fault tolerance and architectures for interconnection networks.

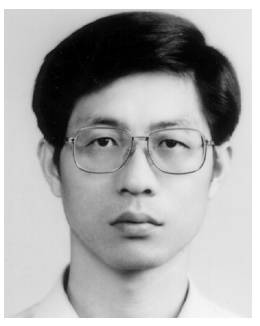

Men-Yang Lin received his MS degree in applied mathematics from National Chung Hsing University in 1986. He is currently a lecturer at the Department of Information Management, National Taichung Institute of Commerce, Taiwan, Republic of China. His main research interests are in graph algorithms and interconnection networks.

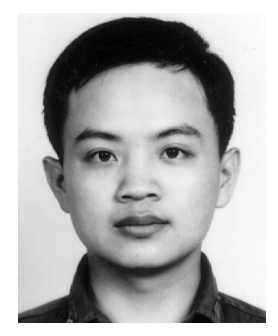

Tung-Yang Ho received the BS and MS degrees in applied mathematics in 1984 and 1989, respectively, and the $\mathrm{PhD}$ degree in computer science in 1995, all from National Chiao Tung University, Taiwan, Republic of China. Currently, he is a post-doctoral researcher at the Institute of Information Science, Academia Sinica, Taiwan, Republic of China. His current research interests are in applications of graph theory to network architectures, CMOS layout, and graph algorithms. 